\title{
Detection of Lithium X-rays by EDS
}

\author{
Li Xiaobing ${ }^{1}$, J. Holland ${ }^{1}$, S. Burgess ${ }^{1}$, S. Bhadare ${ }^{1}$, S. Yamaguchi ${ }^{2}$, D. Birtwistle ${ }^{1}$, P. Statham ${ }^{1}$, and N. \\ Rowlands $^{3}$ \\ 1. Oxford Instruments NanoAnalysis, Halifax Road, High Wycombe, HP12 3SE, UK \\ 2. Oxford Instruments KK, Koto-ku, Tokyo, Japan \\ 3. Oxford Instruments Inc, 300 Baker Ave, Concord, MA 01742, USA
}

Recent advances in hardware technology for EDS have improved detection capability in many areas. Large area detectors with good resolution, made possible by SDD technology, have increased sensitivity so that acceptable count rates can be obtained at the low accelerating voltages. One of the less heralded areas is the progress in the efficiency of the detection of very low energy X-rays. While the detection of Be $\mathrm{K} \alpha$ using $\mathrm{Si}(\mathrm{Li})$ technology in SEM was first reported almost 30 years ago, it was only a few years ago when SDD had progressed sufficiently to detect BeK $\alpha$. However, progress has continued and we are now able to report latest progress in this area. This study focuses on detection and measurement of very low energy X-rays (150eV and below), in particular the detection of LiK $\alpha$ X-rays at $54 \mathrm{eV}$.

Low noise sensor electronics and enhanced charge collection efficiency have improved sensitivity to SiLl at $92 \mathrm{eV}$. The improvements achieved have resulted in clear SiLl and AlLl lines being commonly observed in materials such as Al-Si alloys using $80 \mathrm{~mm}^{2}$ and $150 \mathrm{~mm}^{2}$ area detectors. The other significant barrier to detection is the poor transmission of very low energy X-rays through the detector vacuum window. Even the latest polymer technologies offer little usable transmission below AlLl. To test the potential of the latest sensor package designs, a number of special detectors have been made using a windowless design, to maximize low energy sensitivity.

To test the feasibility of this detector technology for the detection of X-rays from lithium, a sample of Li metal was prepared. The sample was cut from a $\mathrm{Li}$ metal rod in a bag pressurised with pure $\mathrm{N}_{2}$ to minimise surface contamination through reaction with air, before rapid transfer to the load lock of the SEM. A spectrum collected from the sample shown in Fig. A, shows a pronounced peak at around $54 \mathrm{eV}$, well above the X-ray background and clearly separated from the triggered noise peak. Other peaks, particularly $\mathrm{OK} \alpha$, but also $\mathrm{CK} \alpha, \mathrm{NaK} \alpha$ and in some areas $\mathrm{NK} \alpha$ were also detected. The $\mathrm{OK} \alpha$ is always higher than the Li peak, but this is to be expected because the efficiency of the detector at $500 \mathrm{eV}$ is many times that at $50 \mathrm{eV}$, even with this special detector configuration.

To make sure that the observed Li peak was not some artifact of the excitation or detection system, spectra were collected from a number of other materials with no Li content using exactly the same microscope, detector and electronic configuration. No spurious signal was seen in the range $40-70 \mathrm{eV}$ in any spectrum other than that from the Li metal, providing strong evidence that the peak near $54 \mathrm{eV}$ detected in the spectrum from Li metal is indeed due to the LiK $\alpha$ characteristic X-ray emission. Fig. B shows the spectrum obtained from $\mathrm{Cr}$ metal where there is clearly no low energy peak at the LiK $\alpha$ energy.

Li metal is reactive to a number of gases that the sample was exposed to during preparation including $\mathrm{N}_{2}$ to form $\mathrm{Li}_{3} \mathrm{~N}$ and atmospheric water vapour to form $\mathrm{LiOH}$ [1]. Therefore further tests have been conducted on a Li foil sample that was prepared, and loaded through a special microscope loading 
process under vacuum and argon at Hitachi High Technology Corp., Katsuta, Japan. Spectra collected from this sample (Fig. C collected at $2.5 \mathrm{kV}$ ) show significantly lower levels of OK $\alpha$, allowing lower $\mathrm{kV}$ to be used. The benefit of this method was shown by exposing this sample to air for 10 minutes. This exposure results in a rapid alteration to oxygen and carbon containing species, preferentially along grain boundaries, as shown by X-ray maps collected for $\mathrm{LiK} \alpha, \mathrm{CK} \alpha$ and $\mathrm{OK} \alpha$ after exposure (Fig. D).

Reference

[1] U. Wietelmann, R J. Bauer (2000), Lithium and Lithium Compounds. Ullmann's Encyclopedia of Industrial Chemistry, Wiley.

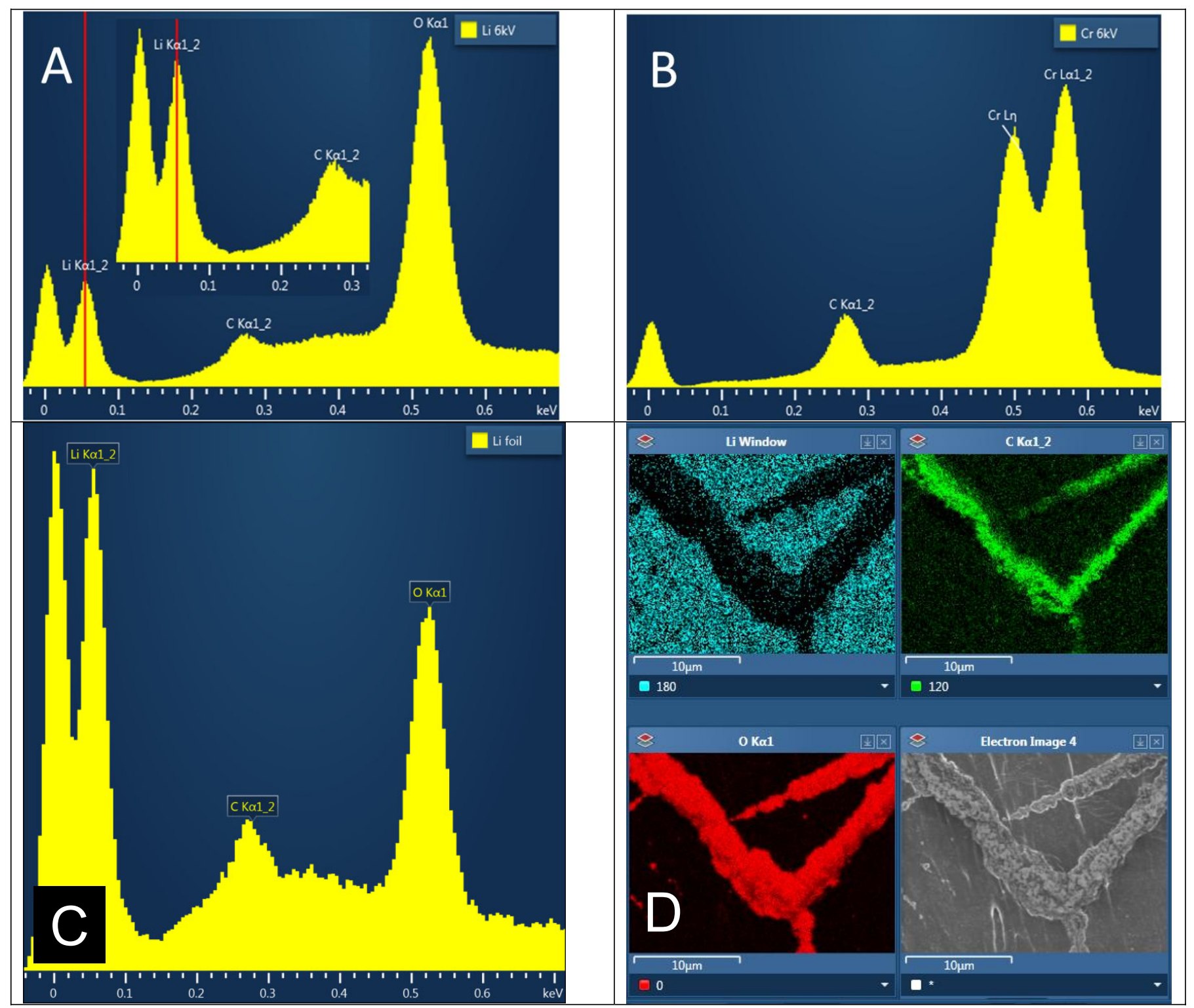

Figs. A. Spectrum collected from corroded lithium metal at $6 \mathrm{kV}$. B. Spectrum collected from chromium metal under identical conditions to the spectrum in Fig. A. C. Spectrum collected from a clean Li foil at $2.5 \mathrm{kV}$. D. X-rays maps for $\mathrm{LiK} \alpha, \mathrm{CKa}$, and $\mathrm{OKa}$ showing grain boundary alteration in the foil after exposure to air. 